Marquette University

e-Publications@Marquette

Poly-p-hydroquinone Ethers: Isoenergetic Molecular Wires with Length-Invariant Oxidation Potentials and Cation Radical Excitation Energies

Maxim Vadimovich Ivanov

Marquette University

Vincent J. Chebny

Marquette University

Marat R. Talipov

Marquette University, marat.talipov@marquette.edu

Rajendra Rathore

Marquette University, rajendra.rathore@marquette.edu

Accepted version. Journal of the American Chemical Society, Vol 139, No. 12 (March 10, 2017): 434-4337. DOI. @ 2017 American Chemical Society. Used with permission. 
Marquette University

e-Publications@Marquette

Chemistry Faculty Research and Publications/College of Arts and Sciences

This paper is NOT THE PUBLISHED VERSION; but the author's final, peer-reviewed manuscript. The published version may be accessed by following the link in the citation below.

Journal of the American Chemical Society, Vol. 139, No. 12 (2017): 4334-4337. DOI. This article is (C) American Chemical Society and permission has been granted for this version to appear in ePublications@Marquette. American Chemical Society does not grant permission for this article to be further copied/distributed or hosted elsewhere without the express permission from American Chemical Society.

\title{
Poly-p-hydroquinone Ethers: Isoenergetic Molecular Wires with Length-Invariant Oxidation Potentials and Cation Radical Excitation Energies
}

\author{
Maxim V. Ivanov \\ Department of Chemistry, Marquette University, Milwaukee, Wisconsin \\ Vincent J. Chebny \\ Department of Chemistry, Marquette University, Milwaukee, Wisconsin \\ Marat R. Talipov \\ Department of Chemistry, Marquette University, Milwaukee, Wisconsin \\ Rajendra Rathore \\ Department of Chemistry, Marquette University, Milwaukee, Wisconsin
}




\section{Abstract}

Typical poly-p-phenylene wires are characterized by strong interchromophoric electronic coupling with redox and optical properties being highly length-dependent. Herein we show that an incorporation of a pair of para-methoxy groups at each $p$-phenylene unit in poly-p-phenylene wires (i.e., $\mathrm{PHE}_{n}$ ) changes the nodal structure of $\mathrm{HOMO}$ that leads to length-invariant oxidation potentials and cation radical excitation energies. As such, $\mathbf{P H E}_{n}$ represents a unique class of isoenergetic wires where hole delocalization mainly occurs via dynamic hopping and thus may serve as an efficient medium for long-range charge transfer. Availability of these wires will allow demonstration of long-range electron transfer via incoherent hopping using donor-bridge-acceptor systems with isoenergetic $\mathbf{P H E}_{n}$-based wires as bridges.

\section{Isoenergetic Wires: length-invariant oxidation and excitation energies}
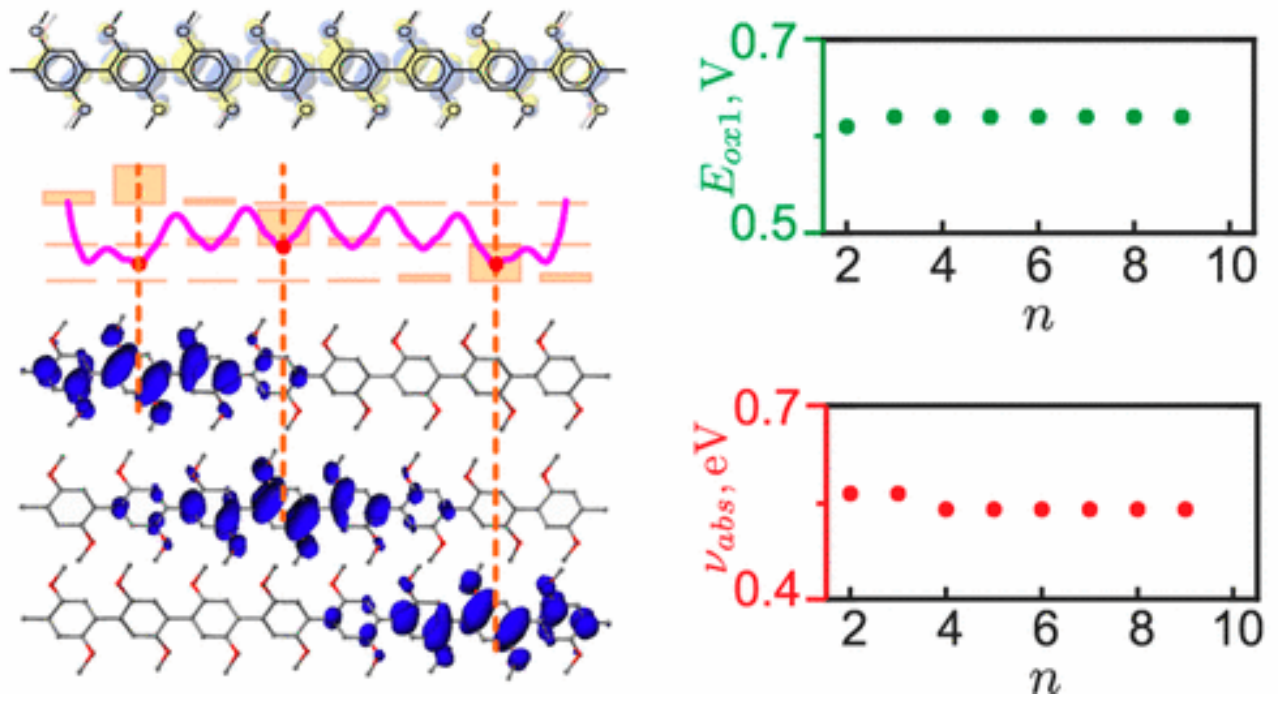

Poly-p-phenylene wires are prototypical materials for long-range charge/energy transfer for potential applications in photovoltaics and molecular electronics. ${ }^{1,2}$ The mechanism of charge transfer in these wires (i.e., tunneling vs hopping) primarily depends on the number of $p$-phenylene units and the degree of interchromophoric electronic coupling, as probed using donor-wire-acceptor and metalwire-metal systems. ${ }^{3-6}$ Typical poly- $p$-phenylene-based wires, such as poly- $p$-phenylenes $\left(\mathbf{P P}_{n}\right)$ or fluorene-based poly- $p$-phenylenes $\left(\mathbf{F P P}_{n}\right)$ are characterized by the strong interchromophoric electronic coupling, due to the favorable nodal (longitudinal) arrangement of HOMO that promotes effective overlap between HOMOs of the monomeric $p$-phenylenes and relatively small dihedral angles (Figure 1).

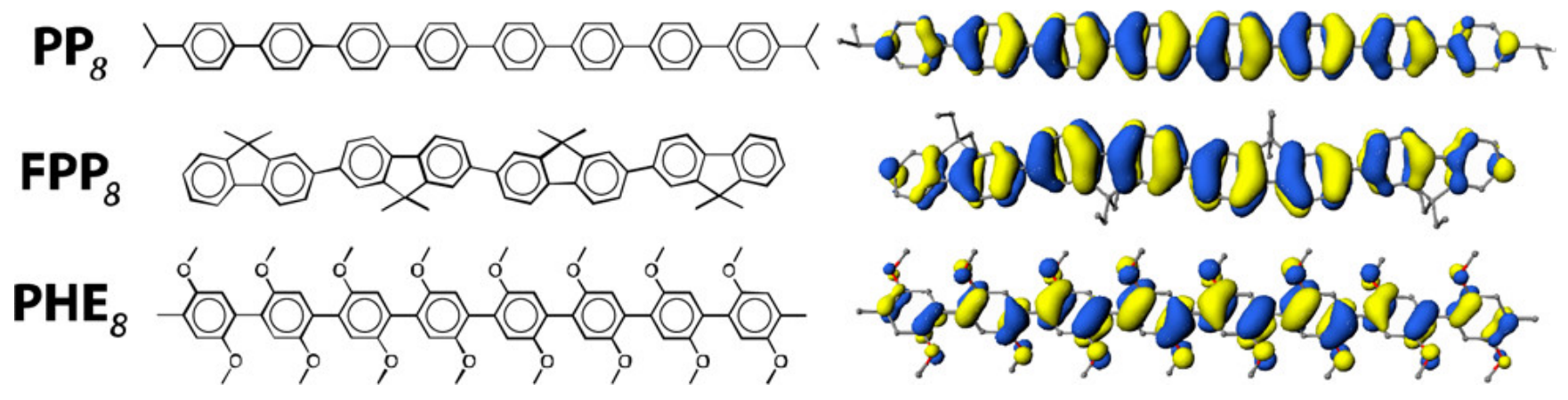

Figure 1. Structures and $\mathrm{HOMOs}$ of $\mathbf{P P}_{8}, \mathrm{FPP}_{8}$ and $\mathbf{P H E}_{8}$.

The electronic absorption energies of neutral $\mathbf{P P}_{n}$ and $\mathbf{F P P} n$ wires follow a linear trend against $\cos [\pi /(n+1)]$ (or $1 / n$ for small $n$ ), in accordance with the Hückel molecular orbital theory. ${ }^{7-10}$ In contrast, their electrochemical oxidation potentials (i.e., $E_{\text {oxi }}$ ), as well as absorption maxima of their 
cation radicals, show an abrupt breakdown from linearity after $n \simeq 8$, indicative of limited hole delocalization. This limited delocalization arises due to the interplay between two factors: (1) the structural/solvent reorganization penalty and (2) the energetic gain from delocalization.

These observations raise an important question: Can the extent of hole delocalization be controlled by modulation of the interchromophoric electronic coupling? Such modulation can in practice be achieved either by altering the interchromophoric dihedral angle ${ }^{11}$ or the nodal structure of the HOMO. ${ }^{12}$ Indeed, placing electron-rich alkoxy groups at 2,5 positions in $\mathbf{P P}_{n}$ wires (i.e., poly- $p$-hydroquinone ethers, $\mathrm{PHE}_{n}$ ) not only alters dihedral angle from $35^{\circ}$ to $56^{\circ}$, but also changes the nodal structure of HOMO from the typical longitudinal arrangement found in $\mathbf{P} \mathbf{P}_{n}$ and $\mathbf{F P P} \mathbf{P}_{n}$ wires to a transverse arrangement (Figure 1).

In this contribution, we will show that the hole delocalization in this new class of $\mathbf{P H E}_{n}{ }^{+\bullet}$ wires, unlike in $\mathbf{P P}_{n}{ }^{+\bullet}$ and $\mathbf{F P P}_{n}{ }^{+\bullet}$, occurs mainly via a dynamic hopping mechanism, leading to length-invariant oxidation potentials and cation radical excitation energies in $\mathrm{PHE}_{n}(n=2-9)$ wires, while their molar absorptivities vary with $n$. This mechanistic distinction was uncovered with the aid of electrochemical analysis, generation of $\mathrm{PHE}_{n}{ }^{+\bullet}$ and measurement of their size-dependent electronic spectra, together with DFT calculations and theoretical modeling. The details of these rather unintuitive yet important findings are described herein.

The highly soluble $\mathrm{PHE}_{n}(n=2-9$ ) wires, decorated with hexyloxy groups, were synthesized using readily available precursors and standard high-yielding reactions such as alkylation of $p$-hydroquinone, bromination, borylation, and Suzuki coupling. Various $\mathrm{PHE}_{n}$ wires were characterized by ${ }^{1} \mathrm{H} /{ }^{13} \mathrm{C} \mathrm{NMR}$ spectroscopy, MALDI mass spectrometry, and X-ray crystallography of representative molecules (see Supporting Information for full details).

The optoelectronic properties of neutral $\mathrm{PHE}_{n}$ wires (i.e., electronic absorption and emission maxima) shift red with increasing number of $p$-hydroquinone ethers units and show a linear dependence against $\cos [\pi /(n+1)]$, similar to those observed for other poly- $p$-phenylenes, such as $\mathbf{P P}_{n}$ and $\mathbf{F P P} \mathbf{P}_{n}$ (see Figures S4 and S5). ${ }^{7}$

Unexpectedly, however, all $\mathrm{PHE}_{n}$ showed reversible cyclic voltammograms (CVs), with invariant redox potential $\left(0.62 \pm 0.01 \mathrm{~V}\right.$ vs $\mathrm{Fc} / \mathrm{Fc}^{+}$) as shown by square wave voltammograms in Figure $2 \mathrm{~A}$ (also see CVs in Figure S6). The invariant oxidation potentials of $\mathbf{P H E}_{n}$ lie in sharp contrast with the observed decrease in oxidation potential in $\mathbf{P P}_{n}$ and $\mathbf{F P P} \mathbf{P}_{n}$ wires with the addition of each successive $p$-phenylene unit (Figure 2B), at least up to 7-8 p-phenylene units. ${ }^{7,9}$ 

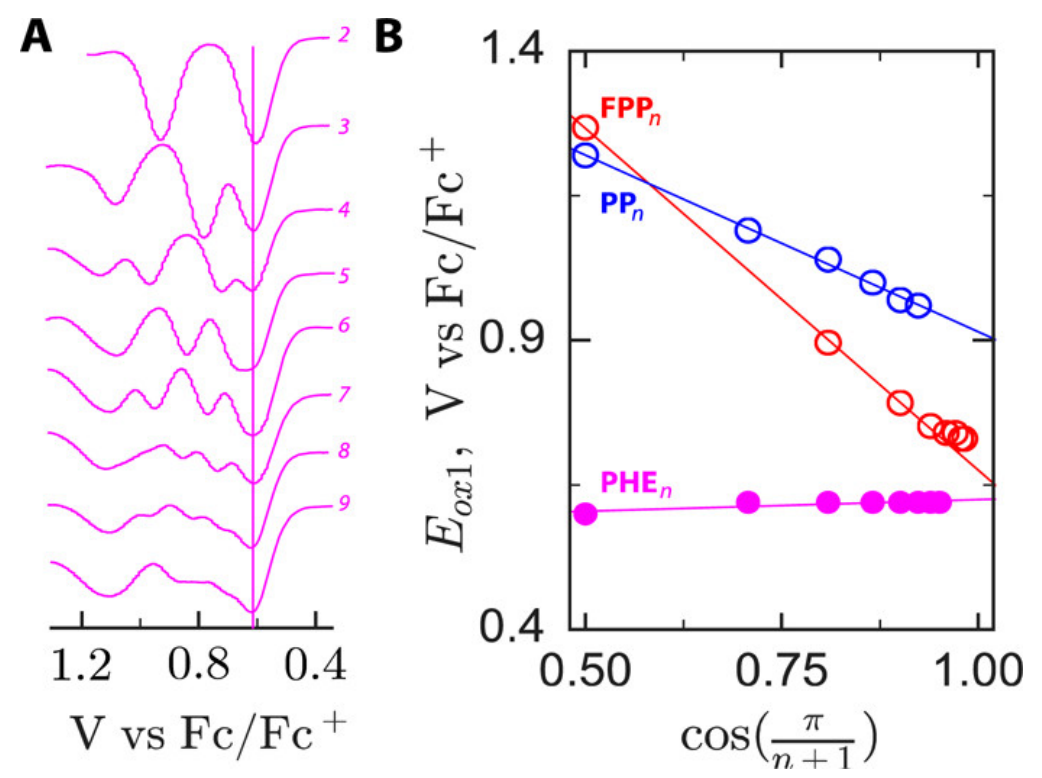

Figure 2. (A) Square-wave voltammograms of $2 \mathrm{mM} \mathrm{PHE}_{n}$ in $\mathrm{CH}_{2} \mathrm{Cl}_{2}\left[0.2 \mathrm{M}\left(n-\mathrm{C}_{4} \mathrm{H}_{9}\right)_{4} \mathrm{NPF}_{6}\right]$ at $22{ }^{\circ} \mathrm{C}$. (B) Plots of $E_{\text {ox1 }}$ of $\mathbf{P H E}_{n}$ (magenta), $\mathbf{P P}_{n}$ (blue), and $\mathbf{F P P}$ (red) against $\cos [\pi /(n+1)]$.

To probe this in further detail, we then generated the cation radicals of $\operatorname{PHE}_{n}(n=2-9)$ via quantitative redox titrations ${ }^{13}$ using a highly robust oxidant [ $\left.\mathrm{THEO}^{+\bullet} \mathrm{SbCl}_{6}{ }^{-}\right]^{14}$ (see Supporting Information for details). The reproducible absorption spectra of $\mathbf{P H E}_{2}{ }^{+\bullet}-\mathbf{P H E}_{9}{ }^{+\bullet}$ thus obtained (Figure $3 \mathrm{~A}$ ) are uncannily similar, revealing similar absorption maxima irrespective of the number of $p$-phenylenes $(2242 \pm 66 \mathrm{~nm}$ or $0.55 \pm 0.02 \mathrm{eV})$. Interestingly, however, the molar absorptivity $(\varepsilon)$ of $\mathbf{P H E}_{2}{ }^{+\bullet}-\mathbf{P H E}_{9}{ }^{+\bullet}$ increases linearly up to $n=5$ and then remains unchanged (Figure 3B). The optoelectronic characteristics of $\mathbf{P H E}_{n}{ }^{+\bullet}$ should be contrasted with $\mathbf{P P}_{n}{ }^{+\bullet}$ and $\mathbf{F P P}{ }_{n}{ }^{+\bullet}$, which vary according to a linear $\cos [\pi /(n+1)]$ trend (Figure 3C). ${ }^{7,9}$
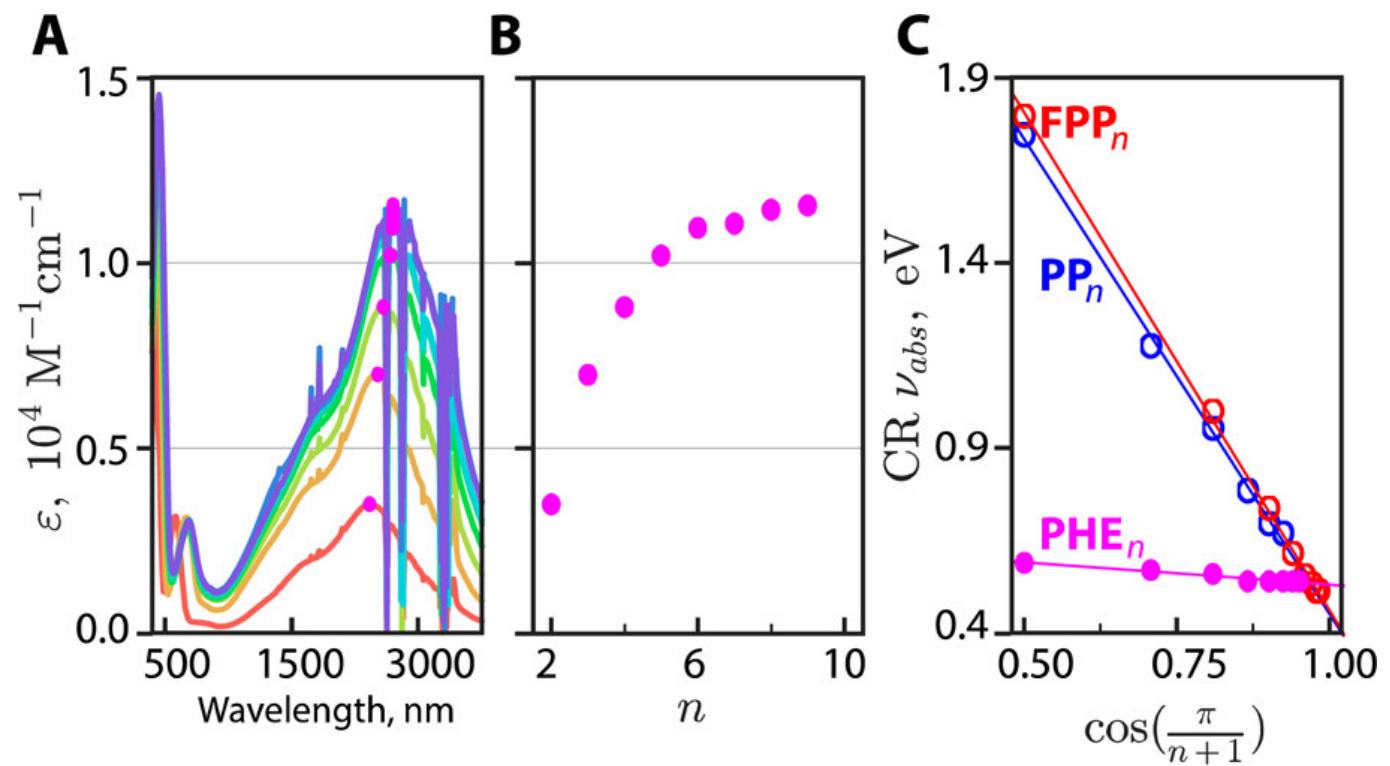

Figure 3. (A) Absorption spectra of $\mathrm{PHE}_{n}{ }^{+\bullet}$ in $\mathrm{CH}_{2} \mathrm{Cl}_{2}$ at $22{ }^{\circ} \mathrm{C}$ against molar absorptivity $(\varepsilon)$. (B) Plot of $\varepsilon_{\max }$ of $\mathbf{P H E}_{n}^{+\bullet}(n=2-9)$ against $n$. (C) Plots of $\mathrm{V}_{\text {abs }}$ of $\mathbf{P H E}_{n}^{+\bullet}$ (magenta), $\mathbf{P P}_{n}^{+\bullet}$ (blue), and $\mathbf{F P P}_{n}^{+\bullet}$ (red) against $\cos [\pi /(n+1)]$ trend.

These observations of the invariant oxidation potentials $\mathbf{P H E}_{n}$ and invariant excitation energies and a linear increase of molar absorptivity up to $n=5$ for $\mathrm{PHE}_{n}{ }^{+\bullet}$ were reproduced by DFT calculations 
[B1LYP-40/6-31G(d)+PCM $\left.\left(\mathrm{CH}_{2} \mathrm{Cl}_{2}\right)\right]$, ${ }^{9}$ see Figure 4A and Supporting Information for details. DFT calculations further showed that the charge/spin distribution is uneven between the two units in $\mathrm{PHE}_{2}{ }^{+\bullet}(66 \% / 34 \%),{ }^{15}$ while in higher homologues $(n \geq 3)$ it is largely limited to 3-4 units, with the most charge ( 70\%) localized at the central unit (Figure $4 B)$.

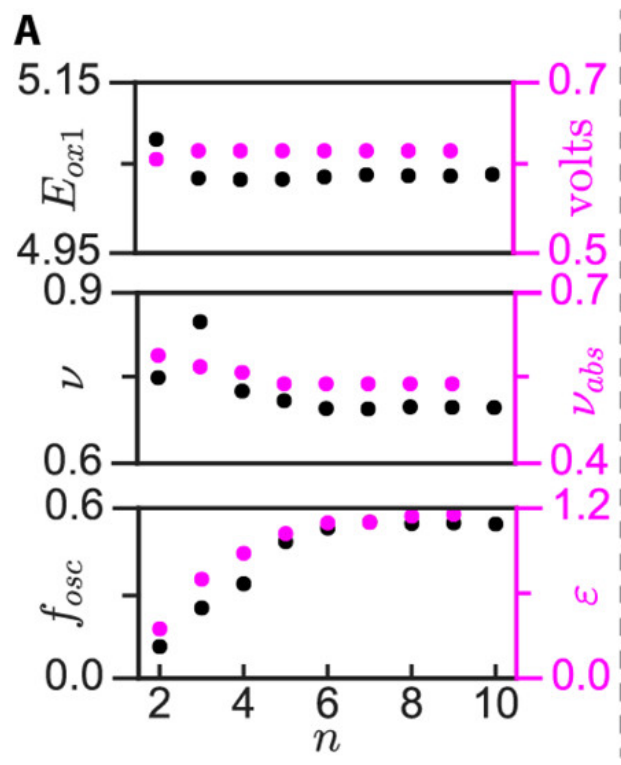

B

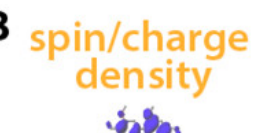
density reorganization
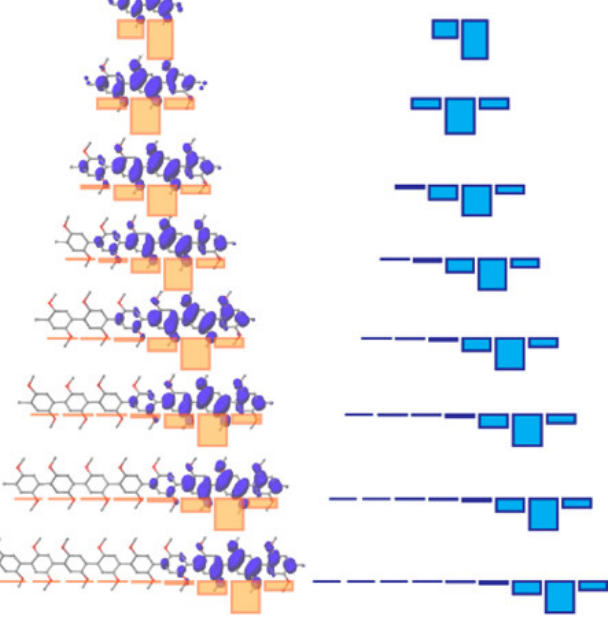

Figure 4. (A) Plots of the calculated (black) oxidation energies (eV, top) of $\mathrm{PHE}_{n}$, and energies of $\mathrm{D}_{0} \rightarrow \mathrm{D}_{1}$ transitions (eV, middle) and oscillator strengths $\left(f_{\text {osc }}\right.$, bottom) of $\mathrm{PHE}_{n}^{+\bullet}$. The experimental points are shown in magenta, i.e., $E_{\mathrm{ox} 1}, v_{\mathrm{abs}}$, and $\varepsilon \times 10^{4} \mathrm{M}^{-1} \mathrm{~cm}^{-1}$. (B) Isovalue plots of spin-densities, per-unit bar plots of the NPA charges/spins (left) and structural reorganization in $\mathrm{PHE}_{n}^{+\bullet}$ (right).

Notably, the spin/charge distribution in $\mathrm{PHE}_{n}{ }^{+\bullet}$ accompanies structural reorganization (compare barplots in Figure 4B), which occurs in the form of quinoidal distortion of each $p$-hydroquinone ether unit. This involves a shortening of the $\mathrm{C}-\mathrm{O}$ bonds (by $\sim 5 \mathrm{pm}$ ), lengthening/contractions (by $\sim 1 \mathrm{pm}$ ) of the equivalent aromatic $\mathrm{C}-\mathrm{C}$ bonds, shortening of the central $\mathrm{C}-\mathrm{C}$ bond (by $\sim 3.2 \mathrm{pm}$ ) and decrease in interplanar dihedral angles between adjacent units $\left(\right.$ by $\left.\sim 17^{\circ}\right)$. Note that the transverse quinoidal distortion in $\mathbf{P H E}_{n}{ }^{+\bullet}$ is contrasted with the longitudinal quinoidal distribution in $\mathbf{P P}_{n}{ }^{+\bullet}$, in accordance with the nodal arrangement of HOMOs, as shown schematically below.
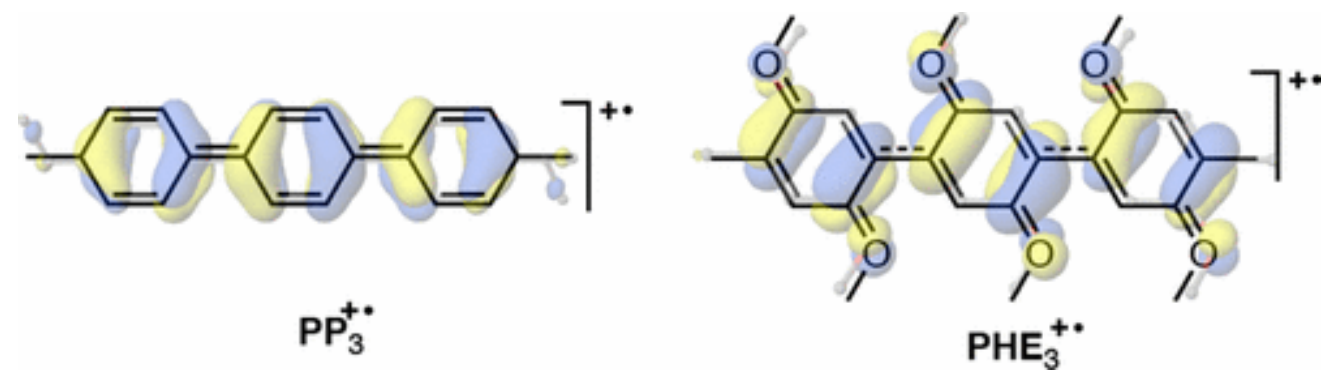

This remarkable difference in nature of the quinoidal distortion is further discerned by X-ray crystallography, which corroborated a much smaller shortening of the central C-C bond in $\mathrm{PHE}_{2}^{+\bullet}$ (by $3.3 \mathrm{pm})(16)$ as compared to typical $\mathbf{P P}_{n}{ }^{+\bullet}$, e.g., a biphenyl (by $\left.4.9 \mathrm{pm}\right)(8)$ and a quaterphenyl (by 5.0 pm). ${ }^{17}$

Our combined DFT/experimental structural analysis amply demonstrates that the mechanism of hole delocalization is different in $\mathbf{P H E}_{n}{ }^{+\bullet}$ and $\mathbf{P P}_{n}{ }^{+\bullet}$. This difference could arise from different 
interchromophoric dihedral angles $\left(37^{\circ}\right.$ vs $\left.56^{\circ}\right)$ as well as different nodal arrangements of the $\mathrm{HOMO}$ (Figure 5A).
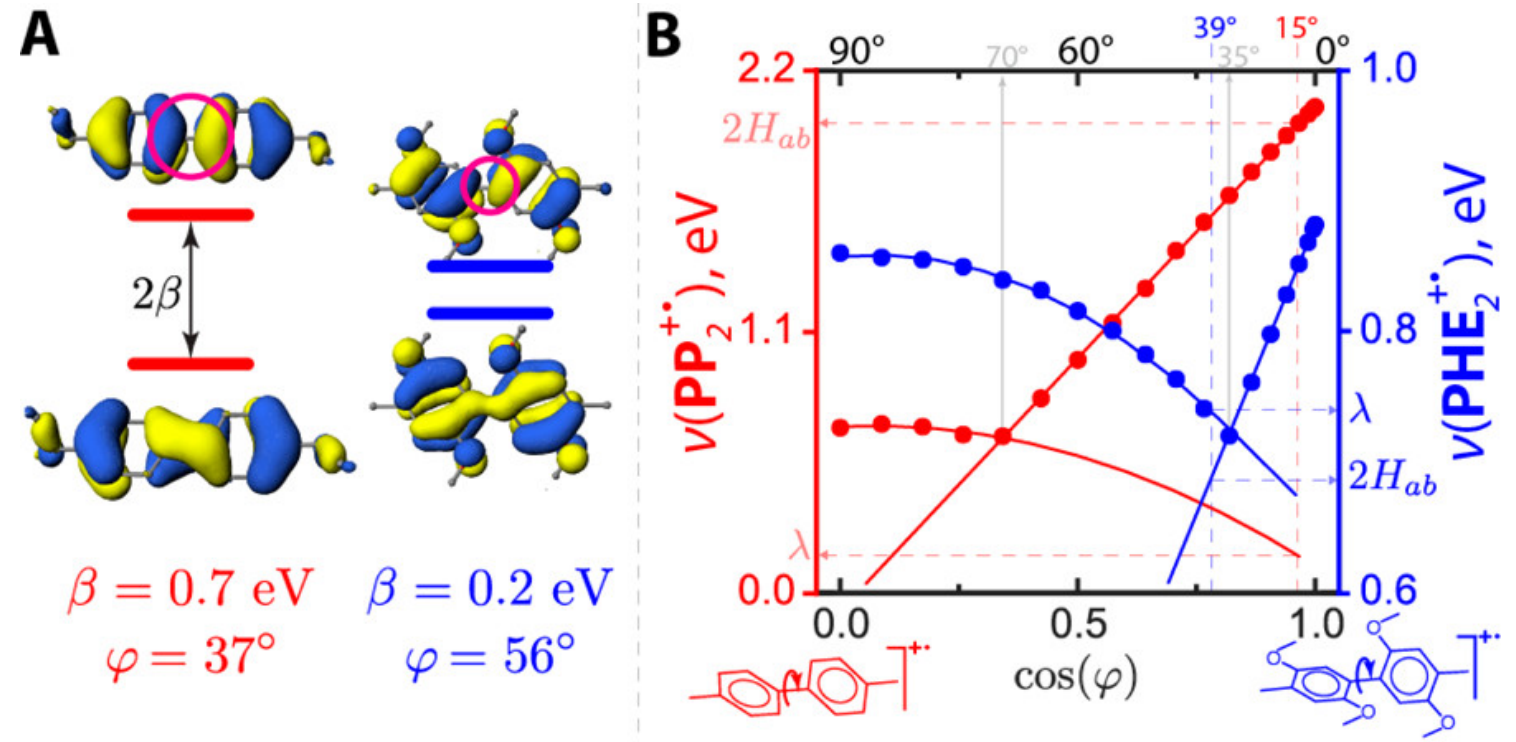

Figure 5. (A) Splitting (2 $\beta$ ) between a pair of bonding and antibonding HOMOs in $\mathbf{P P}_{2}$ and $\mathbf{P H E}_{2}$. (B) Computational scans of $\mathbf{P P}_{2}{ }^{+\bullet}$ (red) and $\mathbf{P H E}_{2}{ }^{+\bullet}$ (blue), obtained as series of constrained optimizations with fixed dihedral angles (at $5^{\circ}$ increment) and subsequent single point TD-DFT calculation to obtain excitation energies $v$. The linear/quadratic fits provided the values of $H_{\mathrm{ab}}$ and $\lambda$ at the equilibirum dihedral angles (see red and blue dotted lines): $H_{\mathrm{ab}}\left(\mathbf{P H E}_{2}{ }^{+\bullet}\right)=0.34 \mathrm{eV}, H_{\mathrm{ab}}\left(\mathbf{P P}_{2}{ }^{+\bullet}\right)=0.99 \mathrm{eV}, \lambda\left(\mathbf{P H E}_{2}{ }^{+\bullet}\right)=0.74 \mathrm{eV}$, and $\lambda\left(\mathbf{P P}_{2}{ }^{+\bullet}\right)=0.16 \mathrm{eV}$.

As shown on the example of $\mathbf{P H E}_{2}$ and $\mathbf{P P}_{2}$ (Figure 5A), incorporation of the methoxy groups changes the nodal arrangement of HOMO from longitudinal to transverse, which results in the reduced orbital overlap between HOMOs of the monomers (red circles in Figure 5A). Thus, both increased dihedral angle and reduced orbital overlap leads to a diminished electronic coupling in $\mathbf{P H E}_{2}(\beta=0.2 \mathrm{eV})$ as compared to $\mathbf{P P}_{2}(\beta=0.7 \mathrm{eV})$. In neutral biaryls, interplanar dihedral angle and orbital overlap controls the electronic coupling, and therefore it is important to consider how these two factors affect the hole delocalization in corresponding cation radicals.

The excitation spectra of the bichromophoric cation radicals are often utilized to estimate electronic coupling $\left(H_{\mathrm{ab}}\right)$ and/or reorganization energy $(\lambda),{ }^{18-22}$ however such estimation of $\lambda$ and $H_{\mathrm{ab}}$ requires $a$ priori knowledge of the mechanism of hole delocalization. We recently demonstrated that the mechanism of hole stabilization in a given biaryl cation radical can be quickly discerned by examining plots of excitation energies $(v)$ versus interchromophoric dihedral angles $(\varphi)$ from $0^{\circ}$ to $90^{\circ}{ }^{11}$ For example, plots of $v$ versus $\cos (\varphi)$ for $\mathbf{P P}_{2}{ }^{+\bullet}$ and $\mathbf{P H E}_{2}{ }^{+\bullet}$ (Figure $5 \mathrm{~B}$ ) show two distinct regimes of static hole delocalization (linear curves) and dynamic hole hopping (quadratic curves) mechanisms. The intersection point of the linear and quadratic curves in Figure 5B represents the crossover angle. ${ }^{11}$

The obtained crossover angles are vastly different for $\mathbf{P P}_{2}^{+\bullet}\left(70^{\circ}\right)$ and $\mathbf{P H E}_{2}{ }^{+\bullet}\left(35^{\circ}\right)$. Thus, the actual equilibrium angle of $15^{\circ}$ in $\mathbf{P P}_{2}{ }^{+\bullet}$ places into a static delocalization regime with the excitation energy providing the electronic coupling (i.e., $\mathrm{v}=2 \mathrm{H}_{\mathrm{ab}}$ ), whereas the dihedral angle of $39^{\circ}$ in $\mathbf{P H E}_{2}{ }^{+}$leads to uneven hole distribution $(70 \% / 30 \%),(15)$ which suggests a dynamic hole hopping mechanism with the excitation energy equal to reorganization energy (i.e., $v=\lambda$ ). Furthermore, extrapolation of the quadratic trend in $\mathbf{P P}_{2}{ }^{+\bullet}$ to the equilibrium angle provides the reorganization energy $\lambda$, while extrapolation of the linear trend to the equilibrium angle of $\mathrm{PHE}_{2}^{+\bullet}$ provides electronic coupling $\mathrm{H}_{\mathrm{ab}}$ (Figure 5B). 
This approach can be further extended to higher homologues of $\mathbf{P H E}_{n}^{+\bullet}$ using our recently developed multistate model $(\operatorname{MSM})(9,23)$-an extension of the Marcus two-state model to several (i.e., $n$ ) chromophoric units. Application of MSM with $H_{\mathrm{ab}}=0.34 \mathrm{eV}$ and $\lambda=0.74 \mathrm{eV}$ together with $\lambda_{\infty}=1.5 \mathrm{eV}$ (that improves excited state description) and $\Delta \varepsilon=-0.13 \mathrm{eV}$ (that accounts for the presence of methyl groups at the terminal units) provides position (i.e., $x_{\min }$ ) and distribution of the hole as well as its oxidation (i.e., $\left.G_{0}\left(x_{\min }\right)\right)$ and excitation (i.e., $\left.\Delta G=G_{1}\left(x_{\min }\right)-G_{0}\left(x_{\min }\right)\right)$ energies via numerical diagonalization of $n \times n$ Hamiltonian matrices for varied charge-transfer coordinate $x$. The redox and optical properties thus obtained by MSM are in excellent agreement with the observed evolution of the experimental data (Figure 6A). In particular, the MSM reproduces the length-independent oxidation $\left(G_{0}\left(x_{\min }\right)=-0.29 \pm 0.01 \mathrm{eV}\right)$ and excitation energies $\left(\Delta G\left(x_{\min }\right)=0.73 \pm 0.04 \mathrm{eV}\right)$ as well as a linear increase of the transition dipole moment $\left(\mu_{01}\right)$ of the $D_{0} \rightarrow D_{1}$ transition up to $n=5$. Note that our earlier application of MSM employed $H_{\mathrm{ab}}$ and $\lambda$ as empirical parameters, which were adjusted to reproduce experimental observables, ${ }^{7,9}$ while in this work these parameters are extracted from the $\mathrm{v} / \cos (\varphi)$ dependence (Figure $5 B$ ).
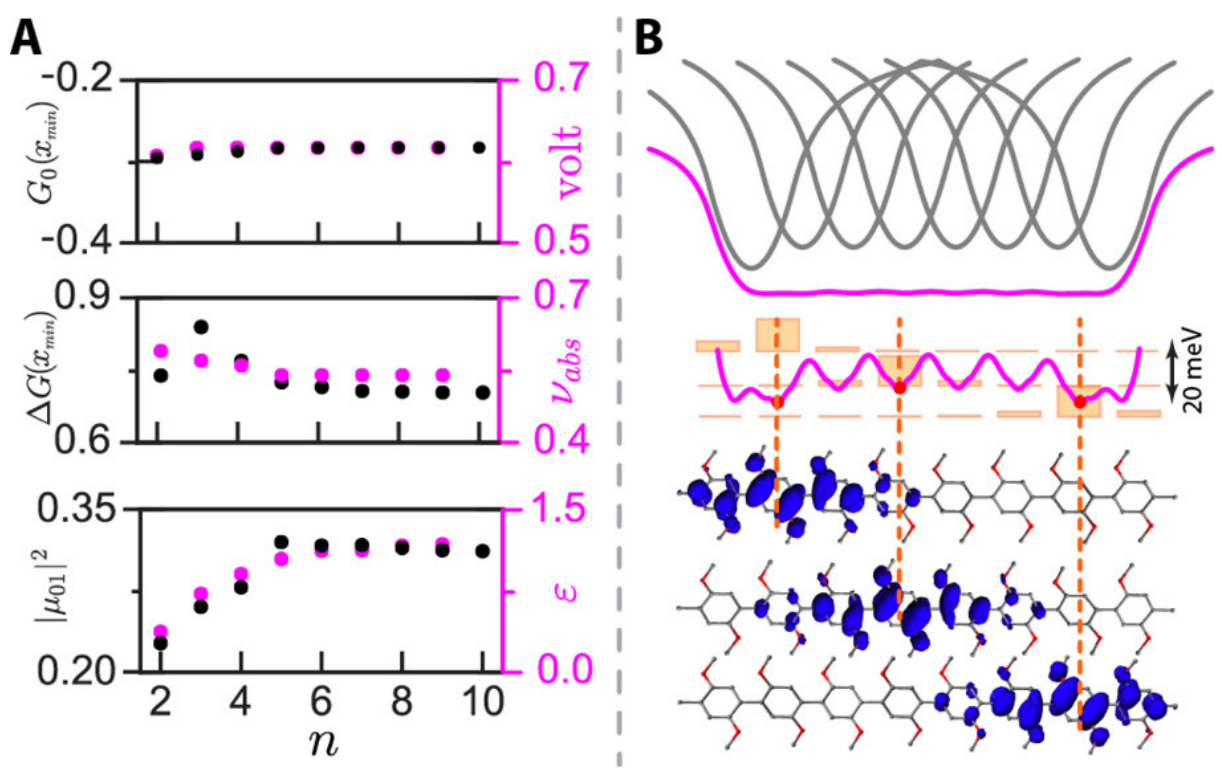

Figure 6. (A) Plots of the oxidation energies $\left(G_{0}\left(x_{\min }\right), e V\right.$, top) of $\mathrm{PHE}_{n}$, excitation energies $\left(\Delta G\left(x_{\min }\right), \mathrm{eV}\right.$, middle) of $\mathrm{PHE}_{n}{ }^{+\bullet}$ and the square of the transition dipole moments $\left(\left|\mu_{01}\right|^{2}\right.$, bottom) and comparison with experimental data (magenta, $E_{\text {ox1 } 1}, V_{a b s}, \varepsilon \times 10^{4} \mathrm{M}^{-1} \mathrm{~cm}^{-1}$ ). (B) Diabatic (gray) and ground-state adiabatic (magenta) surfaces of $\mathrm{PHE}_{8}{ }^{+\bullet}$ (top). A magnified adiabatic potential energy surface of $\mathrm{PHE}_{8}{ }^{+\bullet}$ superimposed with the per-unit barplot representations of the hole distribution from MSM (middle). Isovalue spin-density plots of the $\mathbf{P H E}_{8}{ }^{+\bullet}$ isomers from DFT (bottom).

A zoom-in view of the almost flat ground state potential energy surface of $\operatorname{PHE}_{n}^{+\bullet}(n>4)$ reveals the presence of multiple quasi-isoenergetic minima, which represent different positions of the holebearing units along the chain (Figure 6B). Indeed, DFT calculations confirmed the existence of these isomers of $\mathrm{PHE}_{n}^{+\bullet}(n=5-8)$ which differ in energy by $<0.1 \mathrm{kcal} / \mathrm{mol}$ (Figure 6B). The dynamic interconversion among $\mathbf{P H E}_{n}^{+\bullet}$ isomers with a minimal barrier ( $\sim 0.5 \mathrm{kcal} / \mathrm{mol}$ or $20 \mathrm{meV}$, Figure 6B) should provide an effective platform for incoherent charge transfer along the long poly- $p$ hydroquinone ether wires. ${ }^{24,25}$

In conclusion, systematic study of a series of poly- $p$-hydroquinone ethers ( PHE $\left._{n}, n=2-9\right)$ showed length-invariant oxidation potentials $\left(E_{\text {ox } 1}=0.62 \pm 0.01 \mathrm{~V}\right)$ and cation radical excitation energies $\left(V_{\mathrm{abs}}=\right.$ $0.55 \pm 0.02 \mathrm{eV})$, and molar absorptivities that increase up to $n=5$. These unusual characteristics of 
isoenergetic $\mathrm{PHE}_{n}$ wires arise from an increased interchromophoric dihedral angle and altered nodal arrangement of HOMO due to presence of a pair of methoxy groups at each $p$-phenylene unit, which limits the overlap between the orbitals of monomer units. With the aid of DFT calculations and our recently developed Marcus-based multistate model (MSM) using $H_{\mathrm{ab}}$ and $\lambda$ derived from the $v / \cos (\varphi)$ dependence, ${ }^{11}$ we show that in $\mathrm{PHE}_{n}{ }^{+\bullet}$ hole delocalization occurs mainly by the dynamic hopping mechanism, unlike the static delocalization of the hole over multiple monomeric units in typical poly- $p$ phenylene wires. Despite the observation of limited hole delocalization to 3-4 units in $\mathbf{P H E}_{n}{ }^{+\bullet}$ wires, the presence of multiple (almost isoenergetic) isomers of the $\mathbf{P H E}_{n}{ }^{+\bullet}$ with low interconversion barrier provides a possible pathway for long-range charge transfer in these wires.

\section{Supporting Information}

The Supporting Information is available free of charge on the ACS Publications website at DOI: 10.1021/jacs.7b01226.

\section{Acknowledgement}

We thank the NSF (CHE-1508677) and NIH (R01-HL112639-04) for financial support and Profs. Scott A. Reid and James Gardinier for helpful discussions. Calculations were performed on the Père cluster at Marquette University.

\section{References}

${ }^{1}$ Facchetti, A. Chem. Mater. 2011, 23, 733 DOI: 10.1021/cm102419z

${ }^{2}$ Ratner, M. Nat. Nanotechnol. 2013, 8, 378 DOI: 10.1038/nnano.2013.110

${ }^{3}$ Goldsmith, R. H.; Sinks, L. E.; Kelley, R. F.; Betzen, L. J.; Liu, W.; Weiss, E. A.; Ratner, M. A.; Wasielewski, M. R. Proc. Natl. Acad. Sci. U. S. A. 2005, 102, 3540 DOI: 10.1073/pnas.0408940102

${ }^{4}$ Davis, W. B.; Svec, W. A.; Ratner, M. A.; Wasielewski, M. R. Nature 1998, 396, 60 DOI: 10.1038/23912

${ }^{5}$ Choi, S. H.; Risko, C.; Delgado, M. C. R.; Kim, B.; Bre das, J.-L.; Frisbie, C. D. J. Am. Chem. Soc. 2010, 132, 4358 DOI: 10.1021/ja910547c

${ }^{6}$ Hines, T.; Diez-Perez, I.; Hihath, J.; Liu, H.; Wang, Z.-S.; Zhao, J.; Zhou, G.; Müllen, K.; Tao, N. J. Am. Chem. Soc. 2010, 132, 11658 DOI: 10.1021/ja1040946

7Ivanov, M. V.; Talipov, M. R.; Boddeda, A.; Abdelwahed, S. H.; Rathore, R. J. Phys. Chem. C 2017, 121, 1552 DOI: 10.1021/acs.jpcc.6b12111

${ }^{8}$ Wang, D.; Talipov, M. R.; Ivanov, M. V.; Rathore, R. J. Am. Chem. Soc. 2016, 138, 16337 DOI: 10.1021/jacs.6b09209

${ }^{9}$ Talipov, M. R.; Boddeda, A.; Timerghazin, Q. K.; Rathore, R. J. Phys. Chem. C 2014, 118, 21400 DOI: 10.1021/jp5082752

${ }^{10}$ Larsen, R. E. J. Phys. Chem. C 2016, 120, 9650 DOI: 10.1021/acs.jpcc.6b02138

${ }^{11}$ Talipov, M. R.; Navale, T. S.; Hossain, M. M.; Shukla, R.; Ivanov, M. V.; Rathore, R. Angew. Chem. 2017, 129, 272 DOI: 10.1002/ange.201609695

${ }^{12}$ Talipov, M. R.; Navale, T. S.; Rathore, R. Angew. Chem., Int. Ed. 2015, 54, 14468 DOI: 10.1002/anie.201506402

${ }^{13}$ Talipov, M. R.; Boddeda, A.; Hossain, M. M.; Rathore, R. J. Phys. Org. Chem. 2016, 29, 227 DOI: $10.1002 /$ poc. 3523

${ }^{14}$ Rathore, R.; Burns, C. L.; Deselnicu, M. I. Org. Synth. 2005, 1 DOI: 10.1002/0471264229.0s082.01

${ }^{15} \mathrm{PHE} 2+\bullet$ lies at the borderline between two regimes of hole delocalization where hole distribution can vary drastically upon a little change in Hab; see Figure S29. 
${ }^{16}$ Sun, D.; Lindeman, S. V.; Rathore, R.; Kochi, J. K. J. Chem. Soc. Perkin. Trans. 2. 2001, 1585 DOI: 10.1039/b103139m

${ }^{17}$ Banerjee, M.; Lindeman, S. V.; Rathore, R. J. Am. Chem. Soc. 2007, 129, 8070 DOI: 10.1021/ja0720356

${ }^{18}$ Barnes, J. C.; Fahrenbach, A. C.; Dyar, S. M.; Frasconi, M.; Giesener, M. A.; Zhu, Z.; Liu, Z.; Hartlieb, K. J.; Carmieli, R.; Wasielewski, M. R.; Stoddart, J. F. Proc. Natl. Acad. Sci. U. S. A. 2012, 109, 11546 DOI: 10.1073/pnas.1201561109

${ }^{19}$ Nelsen, S. F. Chem. - Eur. J. 2000, 6, 581 DOI: 10.1002/(SICI)1521-3765(20000218)6:4<581::AIDCHEM581>3.0.CO;2-E

${ }^{20}$ Aly, S. M.; Ho, C.-L.; Fortin, D.; Wong, W.-Y.; Abd-El-Aziz, A. S.; Harvey, P. D. Chem. - Eur. J. 2008, 14, 8341 DOI: 10.1002/chem.200800304

${ }^{21}$ Heckmann, A.; Lambert, C. Angew. Chem., Int. Ed. 2012, 51, 326 DOI: 10.1002/anie.201100944

${ }^{22}$ Brunschwig, B. S.; Creutz, C.; Sutin, N. Chem. Soc. Rev. 2002, 31, 168 DOI: 10.1039/b008034i

${ }^{23}$ Talipov, M. R.; Ivanov, M. V.; Rathore, R. J. Phys. Chem. C 2016, 120, 6402 DOI: 10.1021/acs.jpcc.6b00514

${ }^{24}$ Smith, C. E.; Odoh, S. O.; Ghosh, S.; Gagliardi, L.; Cramer, C. J.; Frisbie, C. D. J. Am. Chem. Soc. 2015, 137, 15732 DOI: 10.1021/jacs.5b07400

${ }^{25}$ Taherinia, D.; Smith, C. E.; Ghosh, S.; Odoh, S. O.; Balhorn, L.; Gagliardi, L.; Cramer, C. J.; Frisbie, C. D. ACS Nano 2016, 10, 4372 DOI: 10.1021/acsnano.5b08126 